Article

\title{
Automatic Descriptor-Based Co-Registration of Frame Hyperspectral Data
}

\author{
Maria Vakalopoulou * and Konstantinos Karantzalos \\ Remote Sensing Laboratory, National Technical University of Athens, \\ Heroon Polytechniou 9, Zographos 15780, Greece; E-Mail: karank@central.ntua.gr \\ * Author to whom correspondence should be addressed; E-Mail: mariavak@ mail.ntua.gr; \\ Tel.: +30-210-7721-673; Fax: +30-210-7722-594.
}

Received: 18 December 2013; in revised form: 19 February 2014 / Accepted: 27 March 2014 / Published: 17 April 2014

\begin{abstract}
Frame hyperspectral sensors, in contrast to push-broom or line-scanning ones, produce hyperspectral datasets with, in general, better geometry but with unregistered spectral bands. Being acquired at different instances and due to platform motion and movements (UAVs, aircrafts, etc.), every spectral band is displaced and acquired with a different geometry. The automatic and accurate registration of hyperspectral datasets from frame sensors remains a challenge. Powerful local feature descriptors when computed over the spectrum fail to extract enough correspondences and successfully complete the registration procedure. To this end, we propose a generic and automated framework which decomposes the problem and enables the efficient computation of a sufficient amount of accurate correspondences over the given spectrum, without using any ancillary data (e.g., from GPS/IMU). First, the spectral bands are divided in spectral groups according to their wavelength. The spectral borders of each group are not strict and their formulation allows certain overlaps. The spectral variance and proximity determine the applicability of every spectral band to act as a reference during the registration procedure. The proposed decomposition allows the descriptor and the robust estimation process to deliver numerous inliers. The search space of possible solutions has been effectively narrowed by sorting and selecting the optimal spectral bands which under an unsupervised manner can quickly recover hypercube's geometry. The developed approach has been qualitatively and quantitatively evaluated with six different datasets obtained by frame sensors onboard aerial platforms and UAVs. Experimental results appear promising.
\end{abstract}


Keywords: earth observation; automation; feature descriptors; affine; UAVs; RANSAC; sensors

\section{Introduction}

Airborne and spaceborne hyperspectral remote sensing offers repetitive, consistent and comprehensive datasets with enhanced discrimination capabilities for the characterization of subtle spectral features and important chemical and physical properties of the observed terrain features/objects. A significant amount of research and development has been concentrated on a variety of applications including environmental monitoring, agricultural, forestry and geological mapping, natural resource exploration, land-use analysis, terrain categorization, water-quality monitoring, etc. [1-3].

Moreover, due to the recent advances on optics and photopic technology, sensors' quality (e.g., signal to noise ratio) is improving continuously and new dual (e.g., [4]) or lightweight ones with low power consumption for unmanned aerial vehicles (UAVs) have been developed and employed (e.g., [5-7]). In all above applications, the continuously and enormously increasing size of acquired data requires the development of efficient operational tools that are able to register accurately and automatically the acquired hypercubes. The registration approach should adaptively vary to accommodate different types of sensors (frame or push-broom) that differ in the acquisition procedure and the resulting geometry.

On the one hand, the push-broom (line scanning) sensors capture gradually (line by line) the spatial image domain and concurrently all spectral bands. Let us denote with $\mathcal{I}: \Omega \subset \mathcal{R}^{3} \rightarrow \mathcal{R}$ a hyperspectral dataset (hypercube), where $\mathcal{I}(x, y, z)=I_{z}(x, y)$ and $x, y$ are the spatial image dimensions and $z$ indexes one of the $N$ spectral bands/channels. Push-broom sensors, at a single moment $t$ and at every sequential acquisition step, capture only one line along the $x$ spatial axis but all the spectral channels across the $z$ spectral axis. Therefore, the acquired hypercube is heavily distorted across the two spatial axis, while all the spectral bands are co-registered. On the other hand, the frame hyperspectral sensors acquire, at a single time instance $t$, spectral information from one channel that covers the whole spatial domain $I(x, y)$. The spectral bands are captured sequentially at consecutive time instances $t$. Therefore, the resulting hypercube is not registered across the $z$ spectral axis. Depending on the movement and motion of the acquisition platform the displacements are in the general case significant, as every image $I_{z}(x, y)$ was not acquired from the same principal point and with the same geometry.

Co-registration of the captured spectral bands is therefore necessary in order to account for the misalignment due to the fact that they were acquired at different time instances. The requirement for registration is further amplified by a number of distortion sources that include, but are not limited to, the movement of the airborne platform upon which the sensor is mounted, the different acquisition settings (e.g., frame rate, integration time, etc.), and the distortions that are induced by the multi-filter optical system. As a consequence, the registration process should be able to cope with transformations that are parametrized by an increased number of degrees of freedom, such as the affine and the polynomial ones [8,9]. 
In this paper, an automated generic co-registration framework for frame hyperspectral sensors is introduced which is based on feature descriptors. The developed approach has been designed to address affine transformations, but it can be easily extended to other transformations including the case of projective or polynomial models. We focus on airborne frame hyperspectral VNIR sensors which record wavelength bands from $400 \mathrm{~nm}$ to $1000 \mathrm{~nm}$ with a $10 \mathrm{~nm}-80 \mathrm{~nm}$ Full Width at Half Maximum (FWHM), which are usually mounted onboard UAVs or manned helicopters and aircrafts. The proposed frameworks exploits the efficiency of local feature detectors in order to effectively detect key points in salient image regions. Aiming for a generic formulation, all the standard feature descriptor parameters like scale octaves, initial smoothing, gaussian blur, nearest neighbor ratio, contrast/edge thresholds, etc. have been left stable.

\subsection{Related Work}

Image registration has been an active research field during the last decade with a key role for numerous critical applications and in various scientific areas. Several registration approaches have been proposed and detailed in the computer vision and remote sensing literature ([10,11] and the references therein). Today, most commercial image registration software allow the user to manually or automatically register two or numerous images from the visible electromagnetic spectrum which are either single panchromatic ones (one spectral band) or RGB ones (three spectral bands). In principle, state-of-the-art algorithms target applications with either strict requirements in precision or in computation performance/speed.

The implemented methodology consists of establishing correspondences between different images through local feature descriptors. The pioneering scale invariant feature transform (SIFT, [12]) delivers high quality features in the literature due to its high descriptive power and robustness to illumination and viewpoint changes [13,14]. The literature is vast ([13,15] and the references therein); one can mention SURF [16], ASIFT [17], LDAHash [18] for similar approaches that perform better or faster. However, despite their success, their detection repeatability is still low. Tuytelaars et al. [13] reports repeatability rates that are bellow $50 \%$ for RGB datasets. Furthermore, the varying radiometric and geometric conditions, that are typical for remote sensing images, cause detectors to fail to deliver accurate and adequate features/correspondences over the spectrum (Figure 1, [19-21]). In Figure 1, one can observe that the incorrect correspondences (outliers) are dominating when descriptors are applied over the entire spectrum (i.e., sensors range) even with a more sensitive parameter setting. Because of the presence of the incorrect correspondences, the images can not be successfully aligned. Two failures cases (Figures 1a,b is shown in Figure 1 where one can easily notice the increased number of unrealistic correspondences.

Hyperspectral data registration is a challenging problem and a significant body of work has been produced in order to cope with the challenges this problem poses. Among the proposed approaches, one may cite the extension of SIFT for multispectral [19,20] and hyperspectral data [22,23], as well as approaches that optimize the SIFT parameters in order to maximize the number of correspondences [21]. 


\subsection{Contributions}

In this paper, we propose a generic and automated framework which can utilize any descriptor without the need to tune its parameters. Note that for example the SIFT performance is controlled by 17 different parameters $[21,24]$ and their optimal tuning (if any) is an extremely complex procedure for hyperspectral data. Moreover, allowing the delivery of more key points (e.g., increasing octaves, decreasing initial smoothing, etc.) and correspondences results, also, to numerous outliers (Figure 1, [21]).

The focus of this work is to keep the computational complexity as low as possible allowing with optimized coding real time applications. Therefore, the image descriptors are not computed over the entire hypercube [23] or the entire projection space [22], but they are computed at severally bands of the hyperspectral data. This is of significant importance if one takes into account that feature point descriptors are already high-dimensional vectors while the vector SIFT formulation multiplies their complexity by the number of the spectral bands.

Figure 1. Outliers dominate when descriptors are applied over the entire spectrum (i.e., sensor's range) even with a more sensitive parameter setting. In particular, for the spectral bands up to approx. $700 \mathrm{~nm}$ the registration process cannot be completed at all. (a) Correspondences between bands at $783 \mathrm{~nm}$ (left) and at $508 \mathrm{~nm}$ (right) based on scale invariant feature transform (SIFT) and RANSAC; (b) Correspondences between bands at $783 \mathrm{~nm}$ (left) and at $657 \mathrm{~nm}$ (right) based on SIFT and RANSAC.

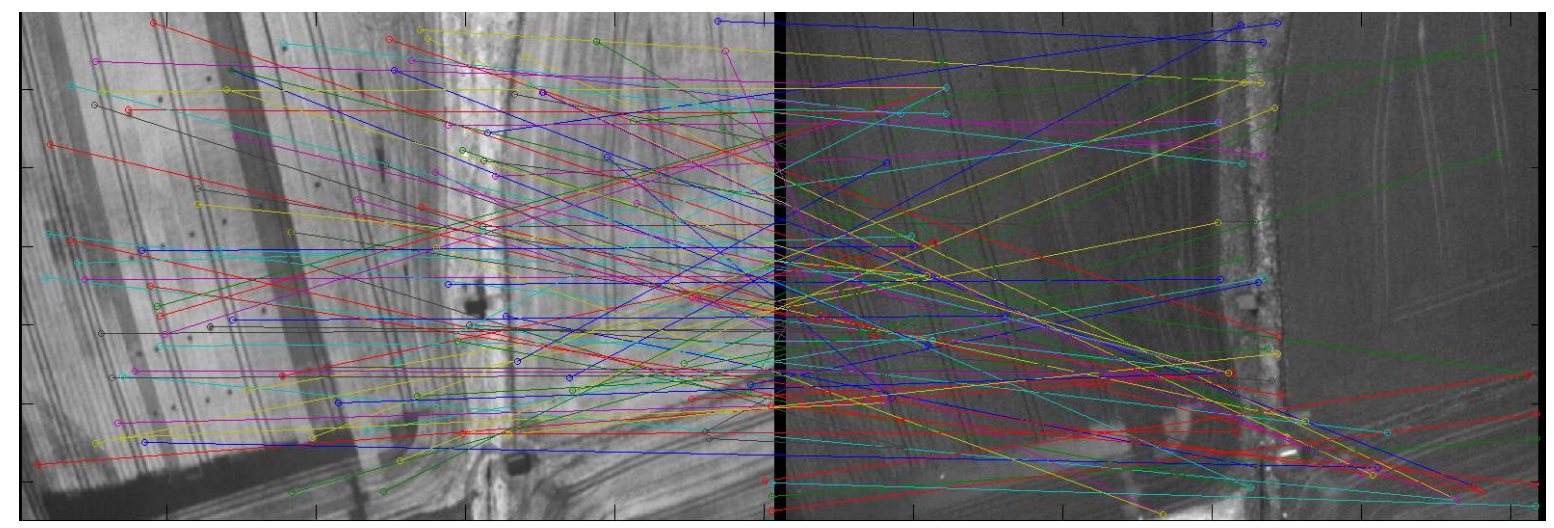

(a)

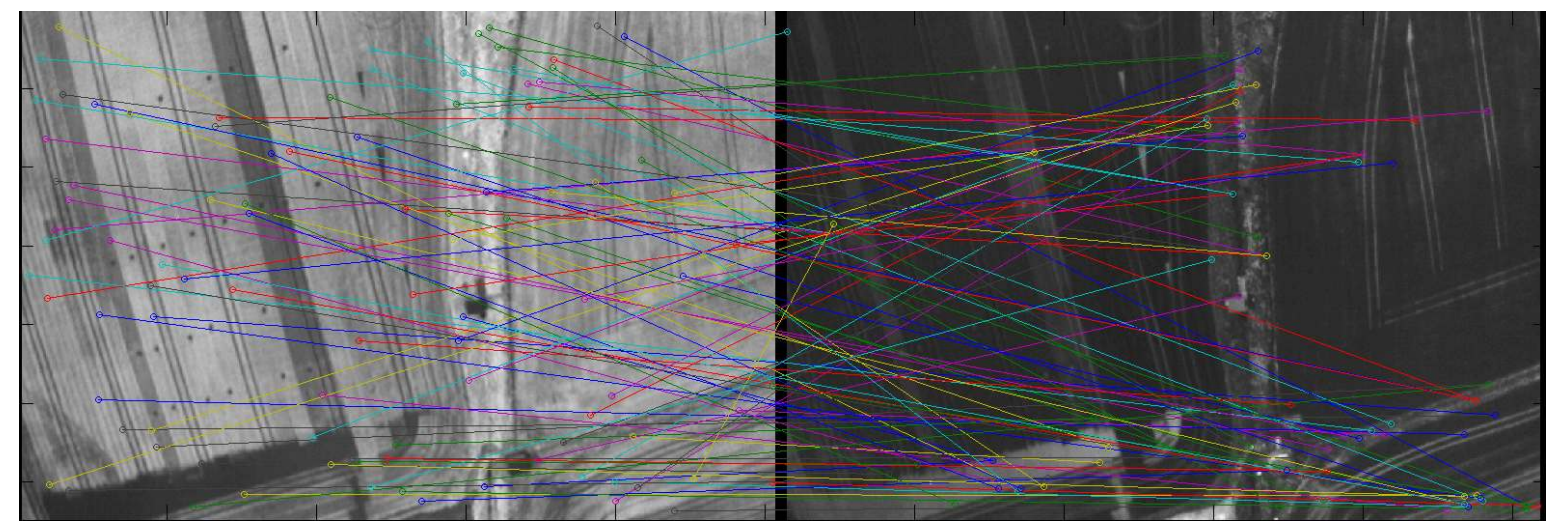

(b) 
Motivated by the observation that the success of automatic feature extraction relies on the descriptor and the characteristics of the input data [13], the proposed approach divides the wavelength in three parts (named Main Spectral Groups, MSG) in which terrain objects usually poses the same stable spectral characteristics and behavior. This allows the efficient decomposition of the registration problem and the accurate fully automated co-registration of every hypercube without using any information e.g., from the GPS/IMU as in similar efforts [7] or propose specific spectral borders as in [7,25]. The algorithm does not make any assumption about the type of sensor, i.e., a Fabry-Perot interferometer with this expected intensity range, radiometric characteristics, etc. Furthermore, generic grouping rules let any dataset from any hyperspectral frame sensor to fit in.

\section{Developed Methodology}

Our goal was to design a generic and automated framework that is able to efficiently register raw hyperspectral data from frame-type sensors. Such data usually contain tens or hundreds of images that in general, depict the same geographic region. However, since every spectral band has been acquired in a different moment, raw data are unregistered. The spatial content of the acquired bands of the hypercube can vary significantly, depending on various parameters like the platform stability, position-hold capabilities, micro-movements, speed, sensor's integration time, etc. In particular, in most cases the overall geometry of each spectral band differs a lot and for example just the translation can be in the range of tens of pixels.

The developed approach is generic, automated and can account for different spatial and spectral resolutions and different spectral ranges, narrow or broader band settings. The developed methodology utilizes data correspondences which have been detected automatically from invariant and robust image descriptors. Throughout our experiments three standard local feature description were employed (namely SIFT, SURF and ASIFT). We compare the three descriptors in terms of efficiency and applicability for frame hyperspectral data from UAVs. Depending on the application one can employ any unsupervised local feature descriptor, robust estimation and transformation/warping algorithm. The developed methodology is able to account for all the spatial and spectral variations and to recover data geometry based on any transformation (like an affine one).

The corresponding flowchart of our implementation is described in Figure 2. More specifically, the developed approach can be divided into the following three processing steps.

\section{(i) Classify spectral bands in Main Spectral Groups (MSG):}

The first step of the procedure is to separate the hypercube into the dominant spectral categories according to the given wavelength (i.e., sensor's sensitivity). The borders of the three main spectral groups were determined based on our experience regarding the representative spectral signatures of the main terrain objects. Water, impervious surfaces/man-made objects and vegetation change, in general, their reflectance/behavior at the green-yellow and red-edge regions. Therefore, and without sticking to a specific sensor or specific wavelenght/channels, three overlapping spectral groups are generated. In particular, for VNIR sensors the borders are defined at the green-yellow $(550-580 \mathrm{~nm})$ and red-edge $(660-700 \mathrm{~nm})$ regions. It should be noted that the borders are not 
exact numerical thresholds but the formulation allows one spectral band to take part in more than one spectral groups. This formulation significantly facilitates the last co-registration step.

Figure 2. The flowchart of the developed algorithm.

\begin{tabular}{|c|c|}
\hline $\begin{array}{c}\text { Divide the dataset in three } \\
\text { Main Spectral Groups (MSG) } \\
\text { group } 1<=580 \mathrm{~nm} \\
\downarrow \\
550 \mathrm{~nm}<\text { group } 2<=700 \mathrm{~nm}\end{array}$ & $\begin{array}{c}\text { Registered Hypercube } \\
\text { find the minimum error } \\
\qquad \downarrow \\
\text { transform MSGs }\end{array}$ \\
\hline group $3>=660 \mathrm{~nm}$ & \\
\hline $\begin{array}{c}\text { Band registration inside every MSG } \\
\text { Choose reference and sort the } \\
\text { spectral bands according to their } \\
\text { spectral radiometric variation } \\
\downarrow \\
\text { run a descriptor } \\
\downarrow \\
\text { run RANSAC } \\
\downarrow \\
\text { calculate affine parameters } \\
\downarrow \\
\text { apply transformations } \\
\downarrow \\
\text { calculate FRE errors }\end{array}$ & $\begin{array}{c}\text { Registration between } \\
\text { the three MSGs } \\
\text { Choose reference and } \\
\text { sort spectral bands } \\
\downarrow \\
\text { run a descriptor } \\
\downarrow \\
\text { run RANSAC } \\
\downarrow \\
\text { calculate affine parameters } \\
\downarrow \\
\text { apply transformations } \\
\downarrow \\
\text { calculate FRE errors }\end{array}$ \\
\hline
\end{tabular}

(ii) Spectral bands registration in each spectral group (MSG):

During the second processing step, the spectral bands from each MSG are co-registered. Unlike similar efforts [7], we do not a priori specify our reference bands. During the initialization process, we calculate the standard deviation of each spectral band as an indication about the spectral/radiometric variation of every channel. In particular, after sorting the bands of each spectral group, the ones with the broader spectral variation are used as a reference and all the other ones from the group are registered to this selected one through an iterative procedure. We select the reference band with the specific unsupervised manner as it makes easier for the detectors to find correspondences with the other bands of the group. One can employ different descriptors (like SURF, SIFT, ASIFT, etc.) and robust estimation procedures (like RANSAC) in order to efficiently detect enough correspondences for the (e.g., affine) transformation and spectral band registration. 
This registration procedure is demonstrated in Figure $3 \mathrm{a}-\mathrm{c}$ for the three corresponding spectral groups in which the developed approach managed to produce enough inliers for the successful in-group registration.

Figure 3. Band registration for every Main Spectral Group (MSG). The calculated registration errors (FRE) were relatively low i.e., from 0.001 to 0.10. (a) Inliers (number of correspondences: 290) during the registration of the 1st spectral group. Bands: 510.2 $\mathrm{nm}$ (left) and $515.4 \mathrm{~nm}$ (right). (b) Inliers (number of correspondences: 72) during the registration of the 2nd spectral group. Bands: $601.7 \mathrm{~nm}$ (left) and $588.6 \mathrm{~nm}$ (right). (c) Inliers (number of correspondences: 75 ) during the registration of the 3rd spectral group. Bands: $783 \mathrm{~nm}$ (left) and $715 \mathrm{~nm}$ (right).

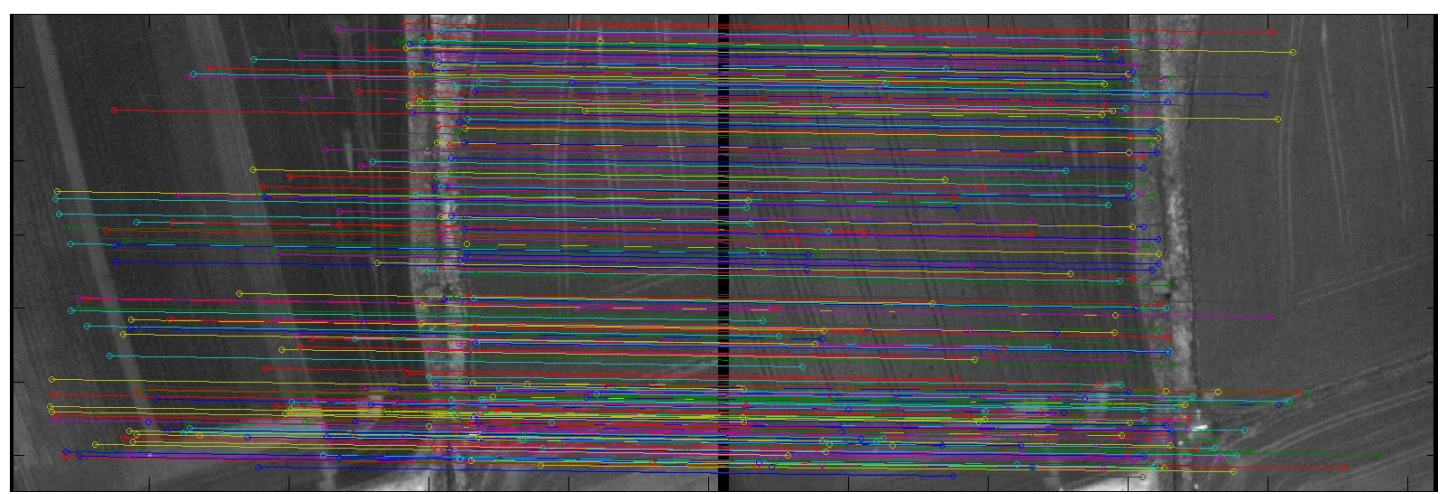

(a)

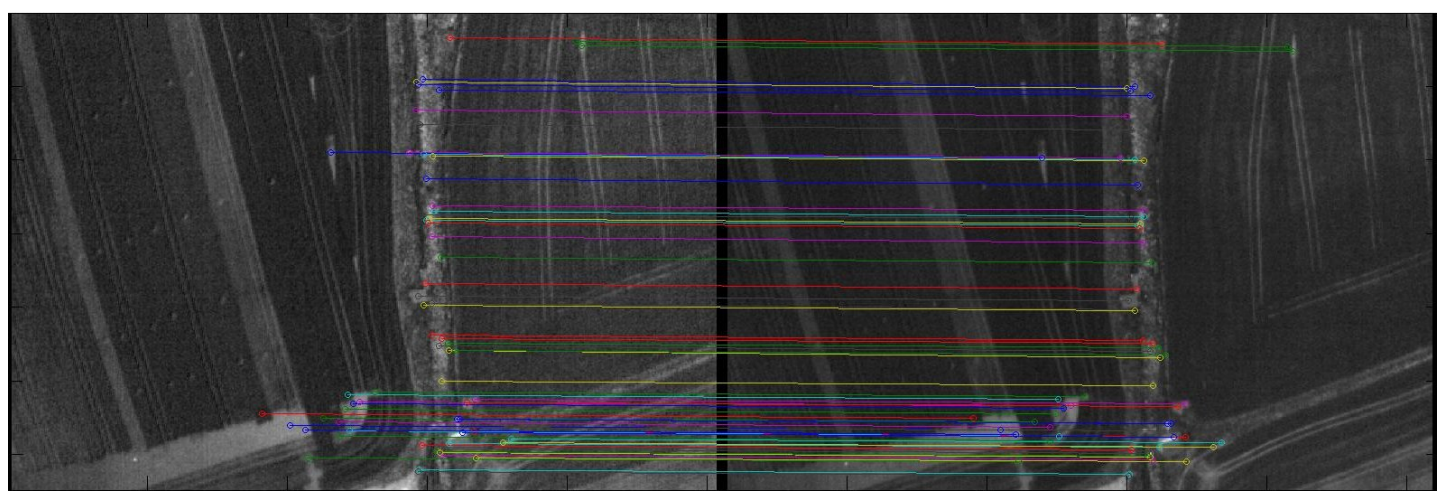

(b)

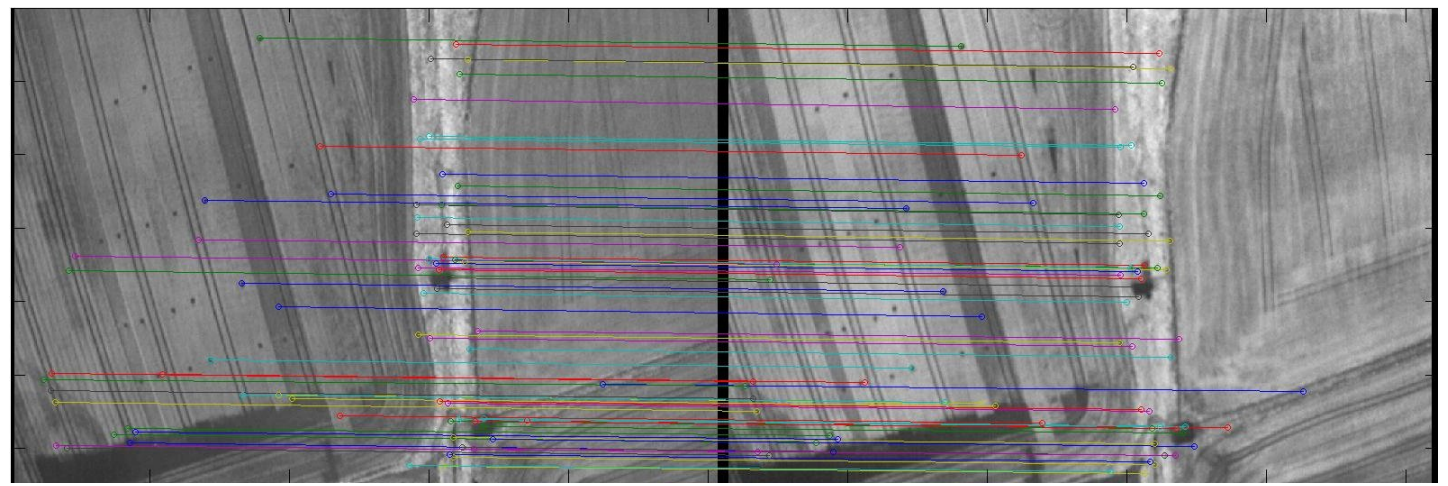

(c) 


\section{(iii) Co-registration of the spectral groups (MSG):}

At this point the spectral bands of each group are registered and the next processing step is to perform the co-registration between the MSGs. Therefore, we propose an iterative approach in order to seek for the appropriate representative bands based on both their spectral variance and proximity to each group. Under such an unsupervised manner the appropriate optimal bands (or series of bands) are selected and correspondences are computed for the co-registration task (e.g., the correspondences between the first and second MSG are shown in Figure 4a while the ones between the first and third shown at Figure 4b). In particular, the algorithm sorts the bands of each group according to their variation (standard deviation) and proximity (spectral distance between the individual bands) in the following manner. First in the list are the bands which served as a reference (larger variation) in the previous step, then follow the bands which are closer to them (with a small spectral distance) and then the rest of the bands according to their standard deviation. This type of sorting enables the convergence usually after just a couple of iterations.

Figure 4. Main Spectral Groups (MSGs) co-registration. The quantitative evaluation (Table 1) resulted in an overall sub-pixel registration accuracy of approximately 0.35 pixels (dataset \# 6). (a) Inliers (number of correspondences: 84) during the co-registration of the 1st and 2nd MSG. Bands: $550.5 \mathrm{~nm}$ (left) and $588.6 \mathrm{~nm}$ (right) (b) Inliers (number of correspondences: 7) during the co-registration of the 2nd and 3rd MSG. Bands: $550.5 \mathrm{~nm}$ (left) and $715.7 \mathrm{~nm}$ (right).

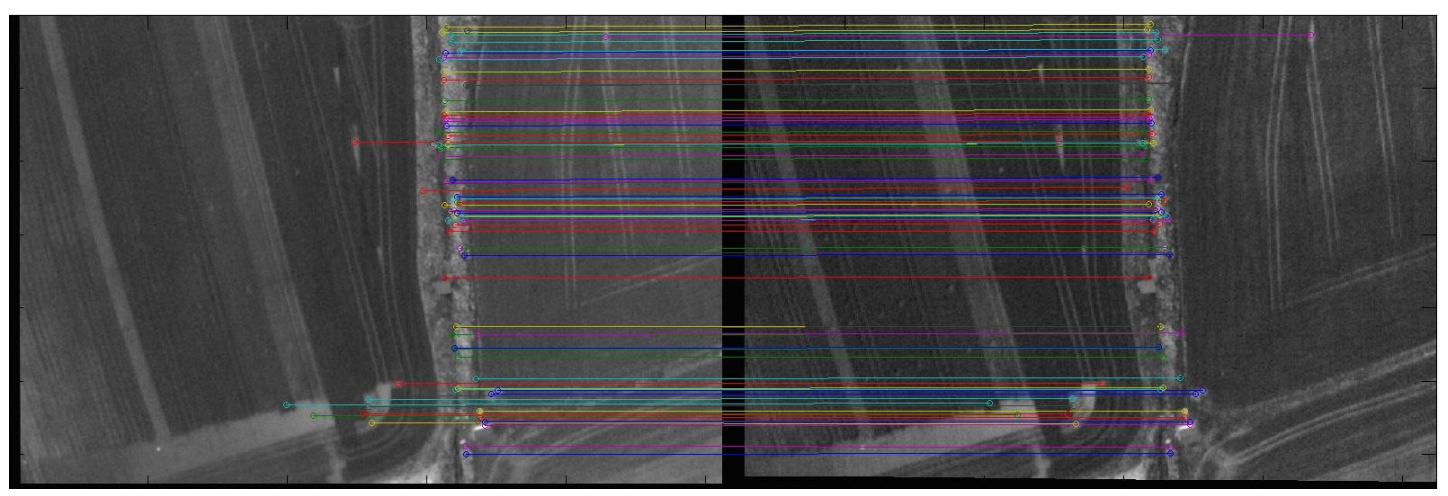

(a)

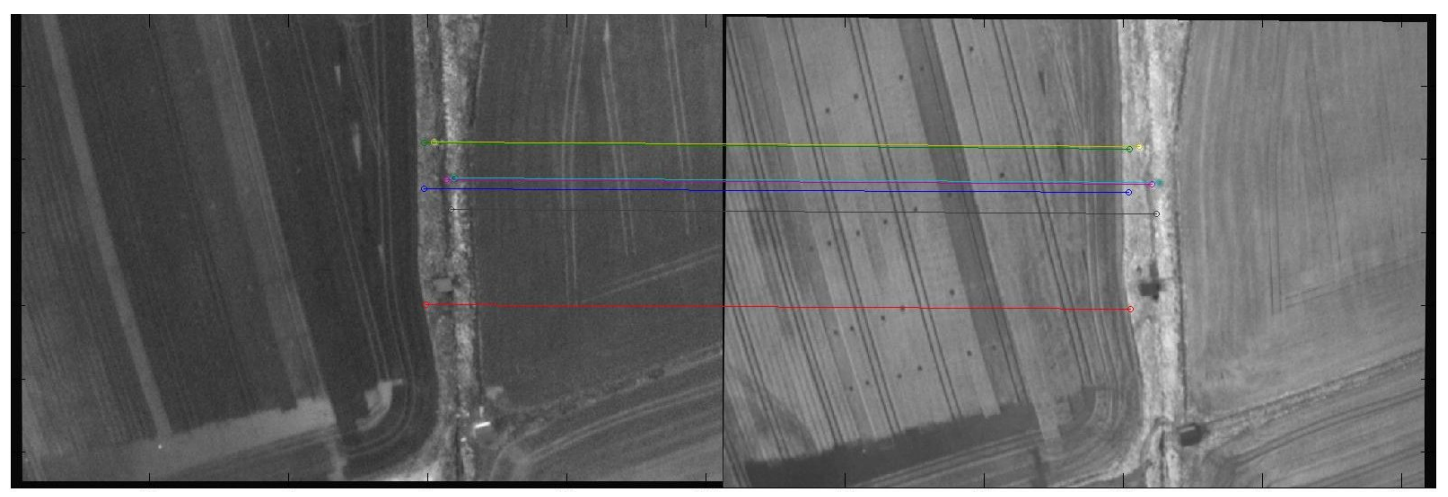

(b) 
Under the proposed formulation we narrow effectively the search space of solutions (image pairs) by selecting in an unsupervised manner the spectral bands which can lead quickly to the required solution. The key values for VNIR sensors are placed near the borders of the green/yellow and the red-edge regions. These regions are defining and determining the band classification in coherent groups. Note that we allow spectral overlaps.

Moreover, in the second and third step, a robust estimation procedure like RANSAC (RANdom SAmple Consensus) is used in order to detect outliers. Throughout our experiments, we account for affine transformations $[X=a(1,1) \times x+a(1,2) \times y+a(1,3) ; Y=a(2,1) \times x+a(2,2) \times y+a(2,3)]$ since it is the model that is commonly used during registration and ortho-rectification procedures. However, other simpler or more complex models can be straight forwardly applied.

In order to evaluate quantitatively the performance of each procedure, the Fiducial Registration Error (FRE) was calculated in every case and for all spectral bands [26]. FRE gives an indication of how well the detected correspondences are matched together. The FRE error is giving an indication of how correct the inliers are, how correct the correspondences serve globally the given transformation. More specifically, FRE is the average ratio calculated by finding the sum difference of all the corresponding points between the reference and the registered image and the sum difference of the reference and the test image.

$$
F R E=\sqrt{\frac{1}{N} \sum_{i=1}^{N} \omega_{i}^{2}\left|R x_{i}+t-y_{i}\right|^{2}}
$$

where $\mathrm{N}$ is the number of correspondences, $\mathrm{R}$ and $\mathrm{t}$ are the parameters of the calculated transformation, $x_{i}$ is the position of the point at the test image, the $y_{i}$ is the position of the point at the reference image and $\omega_{i}$ is a non-negative weighting factor, which may be used to decrease the influence of less reliable points and it is calculated by the formula $\omega_{i}=\frac{1}{\left\langle L E_{i}\right\rangle^{2}}$ where $L E_{i}$ is the difference between the position of the point at the test and reference initial image.

FRE acts as an indicator of the overall registration errors. Briefly, given a certain set of point correspondences, which can be a priori defined, the registration can performed by selecting an appropriate transformation that aligns these point sets (fiducial points or ficucials). In either views of the image, the chosen fiducial points are displaced from their correct locations (localization error, LE).

Through an iterative process and by sorting spectral bands based on the possibility to deliver numerous and correct correspondences across the spectral range of each group, an optimal search path is proposed which can be interrupted as soon as the process reaches an accepted maximum error (FRE). In addition, by allowing spectral overlaps, certain (common) bands are classified in more than one group and therefore in the third step the process may end up to a fully constrained warping i.e., register two groups based on the same spectral band. Therefore, by introducing a generic spectral grouping and by allowing spectral overlaps the possibilities to have a concrete co-registration step are significant. However, in cases that the common bands differ a lot for the group and during the registration procedure large FRE errors are calculated, then they are excluded from the group. 


\section{Experimental Results and Evaluation}

The developed methodology was qualitatively and quantitatively validated on six different datasets. The test data were acquired from a frame hyperspectral sensor onboard a UAV (multicopter and fix-wing). The hyperspectral sensor (Rikola ${ }^{\circledR}$ ) had an horizontal and vertical FOV at $37^{\circ}$ and a default spectral range: $400-1000 \mathrm{~nm}$, with a min spectral resolution (FWHM) at $10 \mathrm{~nm}$. The default spatial dimensions of it are $1024 \times 1024$ pixels with a power consumption less than 5 Watt and a weight less than $600 \mathrm{~g}$. Certain acquired datasets contained 42 spectral bands with a spectral range of 400-850 nm and other from 20 to 40 spectral bands with a spectral range from $450-900 \mathrm{~nm}$. The Rikola sensor is employing a Fabry-Perot interferometer which is formed by two semi transparent mirrors placed face to face. The use of multiple orders of the interferometer is matched to different sensitivities of the RGB pixels of the image sensor. The Fabry-Perot interferometer is placed in the lens system in such a way that the light goes collimated through it and the transmitted spectral bands are a function of the interferometer air gap. By changing the air gap it is possible to acquire a new set of wavelengths for each image. With a sequence of images captured with different air gap values the spectrum is reconstructed for every pixel in the image [27]. The hyperspectral datasets were acquired over different regions consisting of simple and complex landscape, multiple objects of various classes, different texture patterns and an important terrain variability. They also may contain shadows and occlusions.

Firstly, as it is demonstrated in Figure 3 the developed algorithm can address efficiently during the first processing step the registration of every spectral band in every MSG. After deciding the appropriate reference, their co-registration takes place based on the detection of both enough and correct correspondences which is indicated also by the calculated errors. For example, in Figure 3 all spectral bands, including the common ones, have been properly registered with a sufficient amount of accurate correspondences and low registration errors. In particular, bands from the $510.2 \mathrm{~nm}$, $601.7 \mathrm{~nm}$ and $783 \mathrm{~nm}$ were chosen as the reference and the maximum calculated error was (i) 0.02 with 290 correspondences for MSG \#1 as it is demonstrated in Figure 3a; (ii) 0.03 with 72 correspondences for MSG \#2 as shown in Figure $3 b$ and (iii) 0.02 with 75 correspondences for MSG \#3 as shown in Figure 3c.

Furthermore, the third processing step which is addressing the co-registration between the spectral groups has been validated and delivers similar accurate results. Correspondences are computed between the appropriate spectral group representatives and the co-registration of the MSG is performed (Figure 4). This is not a trivial task, however, as the algorithm is seeking for the optimal band combination between different groups that deliver enough correspondences and low errors. Common bands are sorted first in the list and other depending on their spectral variance and proximity follow. For example, in Figure 4a the spectral band at $550.5 \mathrm{~nm}$, which has been successfully registered at both MSGs, was employed in the third processing step resulting to a low registration error of 0.84 with 84 correspondences. The other two MSGs, shown in Figure 4b, were registered based on the spectral bands at $550.5 \mathrm{~nm}$ and $715.7 \mathrm{~nm}$ with a resulting error of 3.24 and when 7 correspondences had been calculated.

Moreover, in Figure 5 one can observe the overall performance of the developed methodology as it has been calculated during the quantitative evaluation. In Figure 5a it is demonstrated that local feature descriptors fail to deliver enough inliers when computed over the entire given spectrum. Both SIFT 
with standard parameter settings and SIFT with a more sensitive setup and more delivered features points fail to calculate effective correspondences and therefore outliers dominate the result. In particular, even with a robust model estimation the registration procedure could not converged for all the bands up to $700 \mathrm{~nm}$, while in contrast the proposed method addressed successfully the entire hypercube. For the default SIFT setup the major parameters were those proposed by Lowe ([12]) i.e., number of octaves: 3, edge threshold: 10 and initial gaussian blur: 1.60. For the sensitive SIFT setup: number of octaves: 6, initial gaussian blur: 1 and nearest neighbor ratio: 0.8. Moreover, regarding the ASIFT ([17]) descriptor the parameters were the same as the default SIFT ones, while the tilt was set to 4 . In a similar way, the SURF ([16]) parameters were the default: number of octaves: 5, initial sampling step: 2 and Hessian response threshold: 0.0002. In general, the standard SIFT and ASIFT techniques failed to detect correspondences between the bands that were just before and after the red edge region (680-700 nm). The SURF and the more sensitive SIFT did find more correspondences, but even after a dual RANSAC application many outliers were dominating the result.

Figure 5. Quantitative Evaluation: (a) Errors for SIFT 2 (standard), SIFT 1 (more sensitive) and the developed (MSG-based) method for the dataset shown in Figure 1 (b) Number of inliers and registration errors (upscaled by a factor of 1000) at certain wavelengths; (c) Mean displacements (error in pixels) before and after the automated registration process.

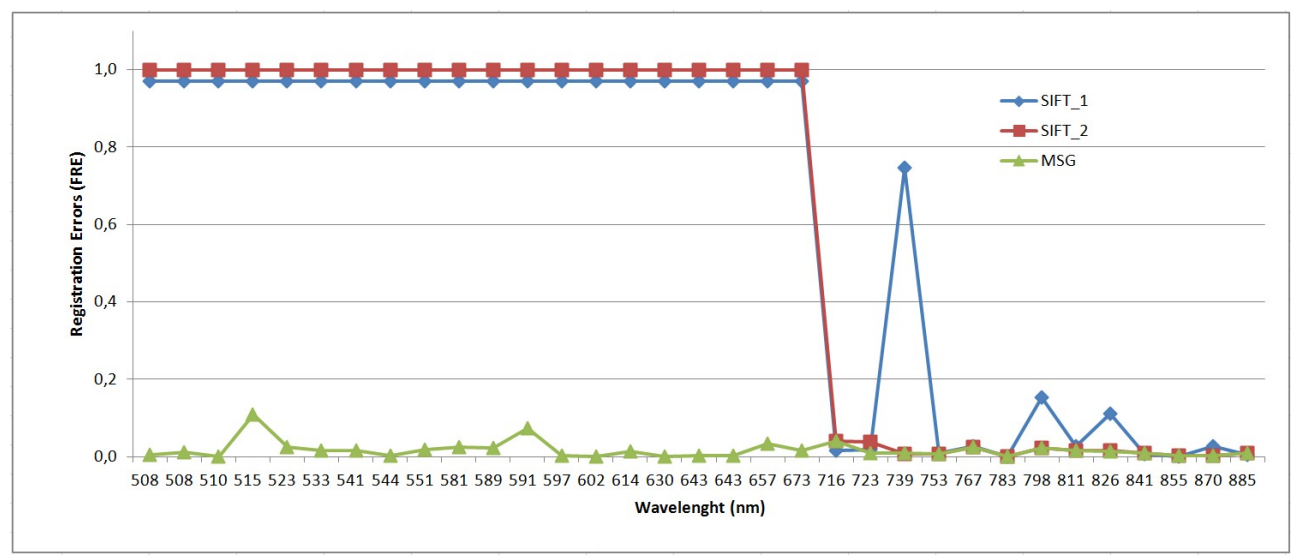

(a)

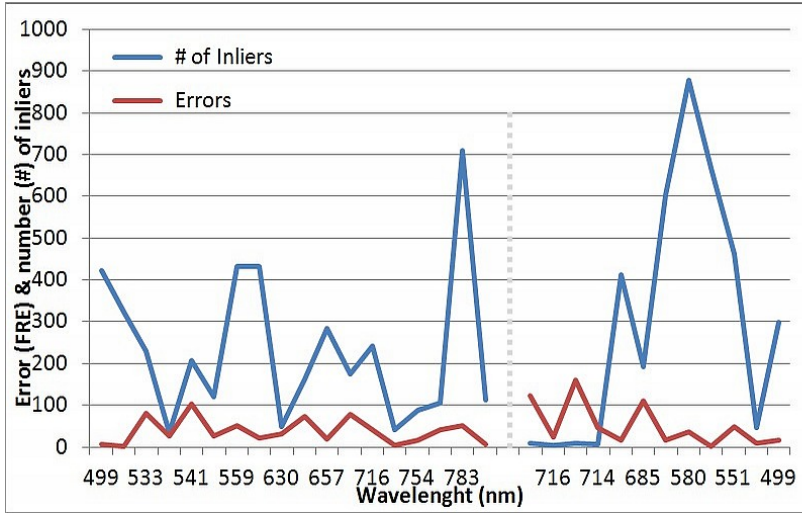

(b)

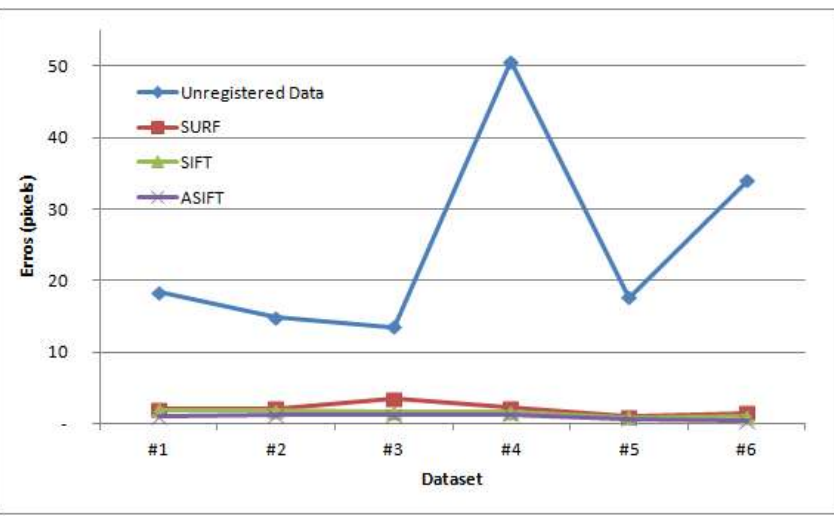

(c) 
In Figure 5b,c it is shown that during the proposed registration procedure the number of resulted inliers is relative large and in all cases sufficient to address the transformation. The calculated errors (in Figure 5b), which are presented for visual reasons upscaled by a factor of 1000, stayed, also, relative low indicating that in all cases the computed correspondences are both enough and correct. Note that the left part of the plot in Figure $5 \mathrm{~b}(499 \mathrm{~nm}-783 \mathrm{~nm})$ indicates the performance of the 2nd processing step and the right part $(716 \mathrm{~nm}-499 \mathrm{~nm})$ the performance of the 3rd processing step. In Figure $5 \mathrm{c}$ the resulting errors, which have been computed from the manually denoted points, of every descriptor for all the datasets are presented (Table 1). The blue line represents the initial mean measured displacement which is more than 13 pixels for all the datasets. The other three lines show the mean errors for every descriptor in all datasets. All descriptors managed to successfully register raw data with a mean displacement error that was lower than 4 pixels. The ASIFT descriptor gave the best results, as the mean displacement error was lower than 1.5 pixels in all datasets. The larger error was calculated when the SURF descriptor was employed over the Dataset \# 3 (3.51 pixels), which depicts an agricultural/rural region and the errors appear mainly on the perimeter of the scene.

Table 1. Quantitative Evaluation: Mean displacements (in pixels) before and after the developed automated registration process. These registration errors have been computed from the manually denoted points which an expert had designated after a careful photointerpretation in every spectral band.

\begin{tabular}{ccccc}
\hline \multirow{2}{*}{ Datasets } & \multirow{2}{*}{ Initial Errors (pixels) } & \multicolumn{3}{c}{ Registration Errors (pixels) } \\
\cline { 3 - 5 } & & SURF & SIFT & ASIFT \\
\hline$\# 1$ & 18.39 & 2.03 & 1.88 & 1.00 \\
$\# 2$ & 14.82 & 2.09 & 1.84 & 1.25 \\
$\# 3$ & 13.50 & 3.51 & 1.57 & 1.17 \\
$\# 4$ & 50.61 & 2.28 & 1.60 & 1.33 \\
$\#$ 5 & 17.60 & 0.98 & 0.88 & 0.66 \\
$\# 6$ & 33.98 & 1.50 & 0.99 & 0.35 \\
\hline
\end{tabular}

In terms of computational performance, the SIFT detector is the fastest one and right after follows the SURF. In all datasets (both agriculture and peri-urban regions) the entire registration process was completed in a few minutes (from $5 \mathrm{~min}$ to $8 \mathrm{~min}$ ) with the SIFT detector, in 10 to 15 min with the SURF detector and it needed up 30 to 90 min when the ASIFT descriptor was employed. In Figures 6-8 one can observe the important spatial variability of raw data. By selecting randomly spectral bands from the raw unregistered hypercube and putting them together in different sub-regions a checkerboard image demonstrates, in the left part of each figure's the initial displacements. Raw unregistered data (e.g., also provided in zoom at lower rows) possess a significant spatial variation with displacements of more than a few pixels. In particular, the initial displacements of raw data are shown in Table 1 where the errors have been calculated by selecting manually the same discrete points in all spectral bands. The registration errors, presented in Table 1, serve as a further quantitative evaluation component. They have been computed from the manually denoted points which an expert had designated after a careful photo-interpretation in every spectral band. 
More specifically, the second dataset (Figure 6) which is over an agricultural region had significant initial displacements which are shown in Figure 6a,c. After the application of the developed algorithm all the spectral bands aligned (Figure 6b,d) and the quantitative evaluation indicated an overall registration error of 1.25 pixels. The registration procedure, under an unsupervised manner, employed the same spectral band at $550 \mathrm{~nm}$ (with 1405 correspondences) for the registration of the first two spectral groups and the spectral bands at $544 \mathrm{~nm}$ and $715 \mathrm{~nm}$, with 10 correspondences, for the registration of the first and third group. The algorithm converged after about 30 min using the ASIFT descriptor and less than 15 and 8 min for the SURF and SIFT detectors, respectively.

Figure 6. Registration results after the application of the developed methodology. The quantitative evaluation (Table 1) calculated an overall accuracy of approximately 1.25 pixels (dataset \# 2). (a) Raw Unregistered Data; (b) Registered Data; (c) Zoom at Raw Data; (d) Zoom at Registered Data.

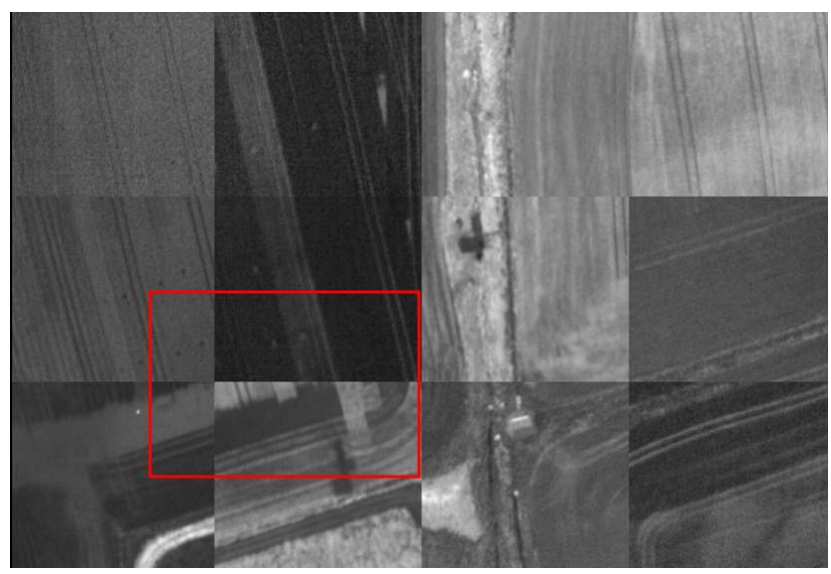

(a)

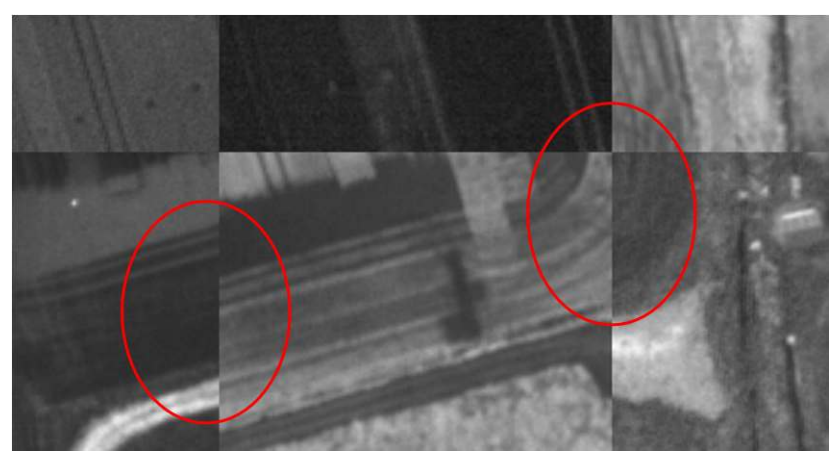

(c)

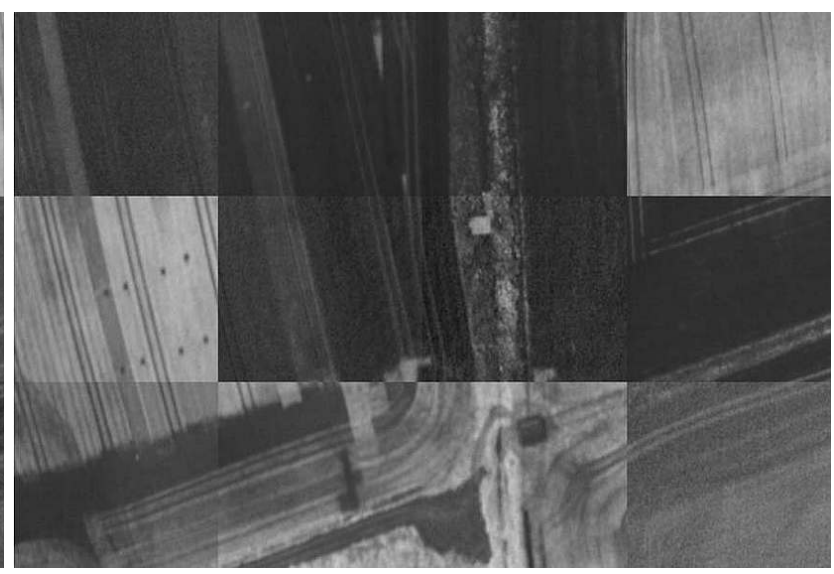

(b)

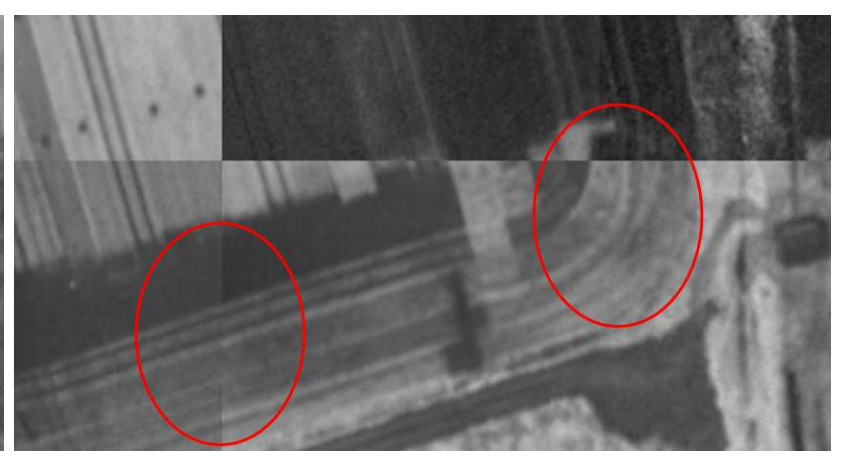

(d)

In Figure 7 the raw and resulting registered hypercubes of Dataset \#5 are shown over a peri-urban region. The acquired from a UAV raw data possessed an important spatial and geometric variation in all spectral bands as it can be observed in Figure 7a,c. The successful application of the developed automated co-registration algorithm managed to recover effectively the geometry of the scene and register all spectral bands successfully (Figure 7b,d). Quantitative evaluation results indicated a sub-pixel accuracy of 0.66 pixels (Table 1). Under an unsupervised manner the procedure selected the common 
spectral band at $559 \mathrm{~nm}$ for the registration of the first and second group with 4286 correspondences and bands at $559 \mathrm{~nm}$ and $685 \mathrm{~nm}$ for the registration of the first and third group (with 2486 correspondences).

With a sub-pixel accuracy were, also, recovered the raw data from Dataset \#6. In Figure 8a,c one can observe the important initial displacements of over 33 pixels. The developed algorithm managed to register all spectral bands and recover their geometry (Figure 8b,d). The resulting overall registration error (Table 1) was 0.35 pixels while the spectral bands at $580 \mathrm{~nm}$ and $685 \mathrm{~nm}$ were the key ones during the third processing step where 4117 correspondences were calculated for the first and second group and 1583 ones for the first and third group.

Figure 7. Registration results after the application of the developed methodology. The quantitative evaluation (Table 1) indicate an overall sub-pixel accuracy of approximately 0.66 pixels (dataset \# 5) (a) Raw Unregistered Data]; (b) Registered Data; (c) Zoom at Raw Data; (d) Zoom at Registered Data.

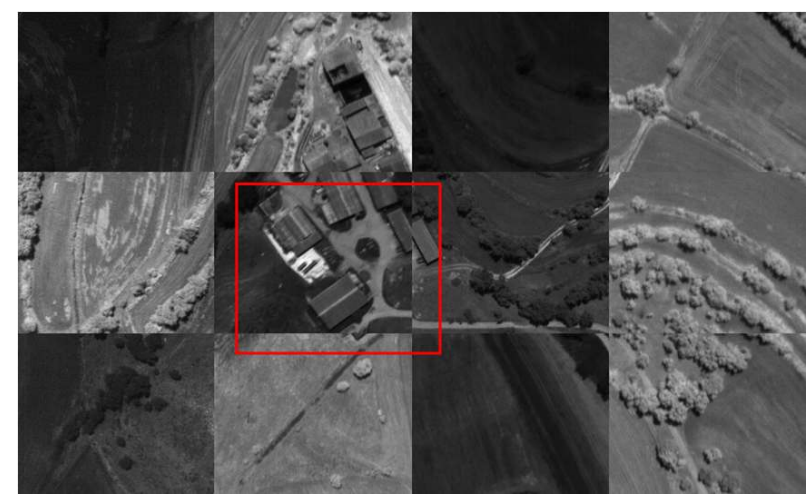

(a)

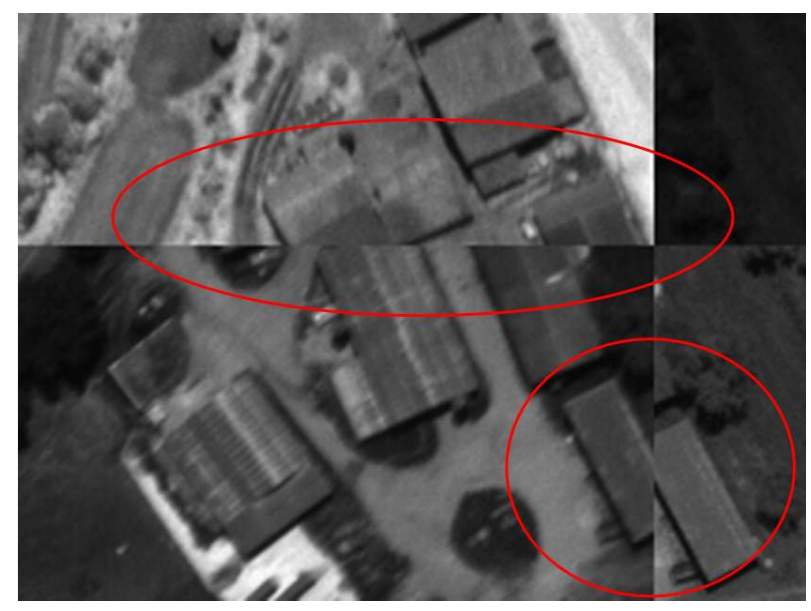

(c)

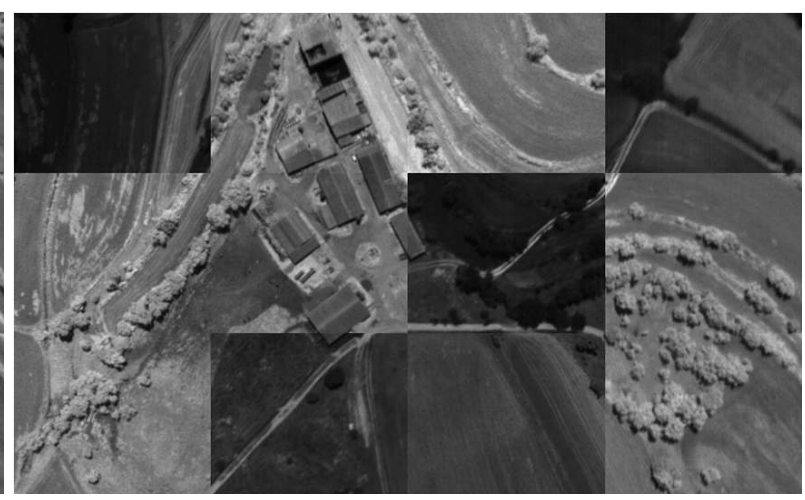

(b)

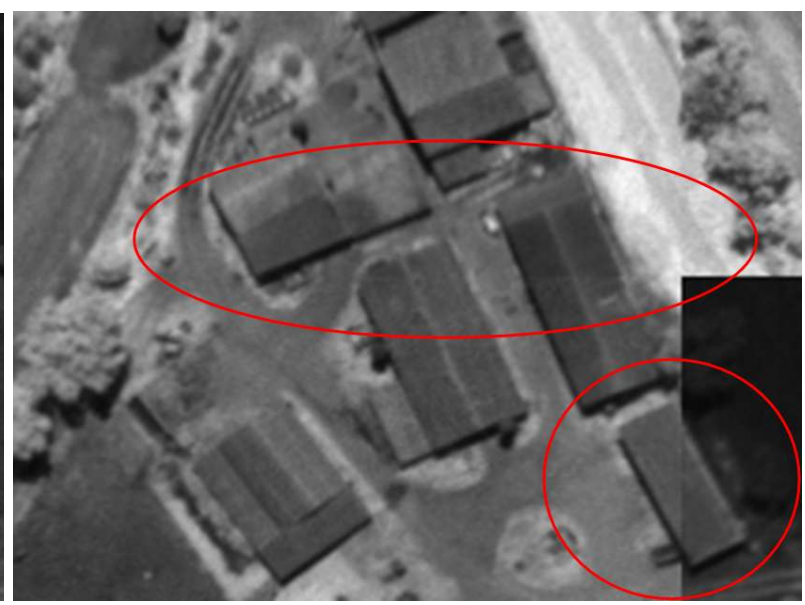

(d) 
Figure 8. Registration results after the application of the developed methodology. The quantitative evaluation (Table 1) indicate an overall sub-pixel accuracy of approximately 0.35 pixels (dataset \# 6) (a) Raw Unregistered Data; (b) Registered Data; (c) Zoom at Raw Data; (d) Zoom at Registered Data.

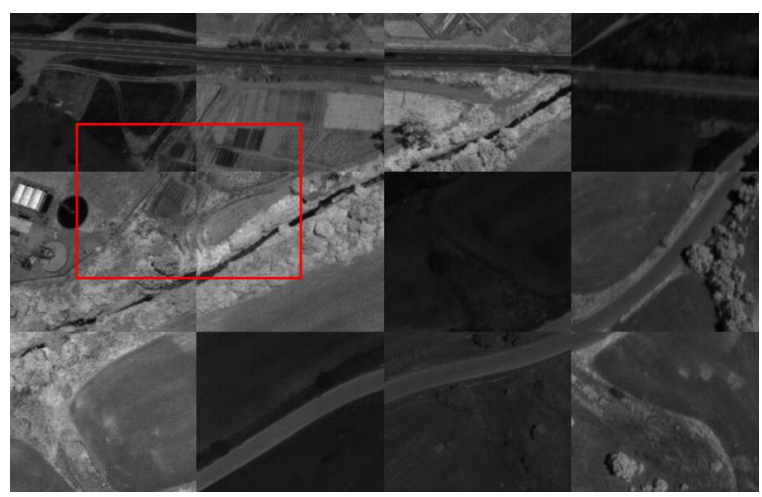

(a)

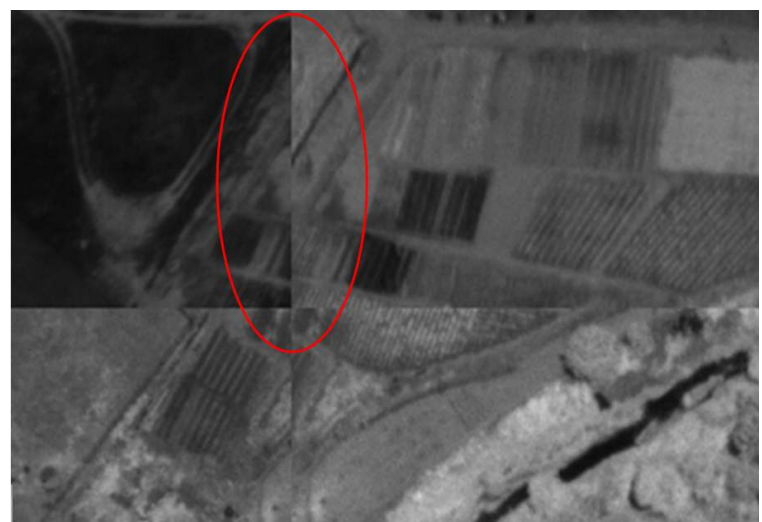

(c)

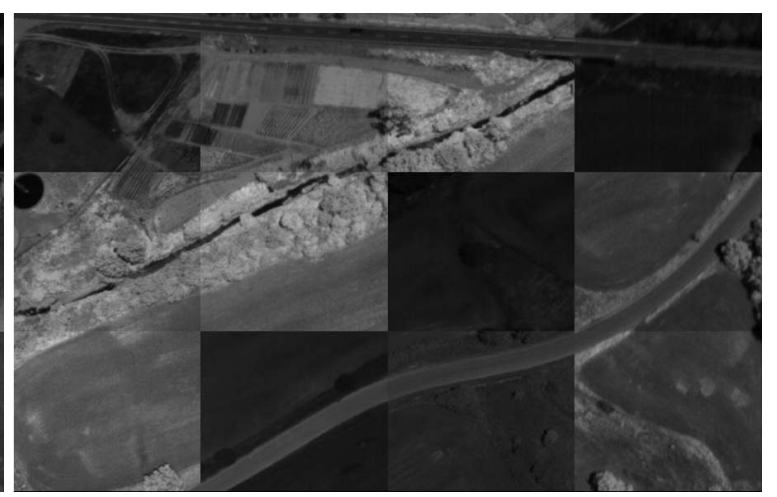

(b)

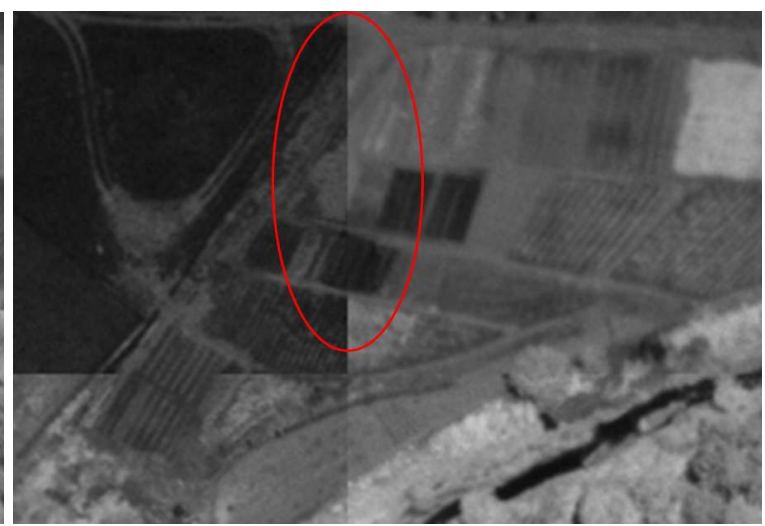

(d)

\section{Conclusions and Future Perspectives}

In this paper, an algorithm for the automatic registration of frame hyperspectral data has been developed and evaluated. The algorithm is relatively fast and efficient. It is based on feature descriptors and the decomposition of the problem into three basic processing steps. The operated wavelength is divided in an appropriate number of spectral groups. Then the co-registration of all the spectral bands of each group is performed. In particular, after sorting the spectral bands in terms of spectral variance and proximity to the key changing spectral regions, the appropriate bands are selected for the co-registration inside and in-between the MSGs. We narrow effectively the search space of solutions by selecting in an unsupervised manner the spectral bands which can lead quickly to the solution. Even with much illumination changes, rotation and translation effects, the image descriptors can find enough correspondences and result to an acceptable accuracy. In all experimental results with different descriptors the algorithm rapidly converged with low errors. The ASIFT descriptor resulted into the best spatial accuracy by delivering more inliers but at the same time was the slowest one. On the other hand, the standard SIFT descriptor detected less inliers with relative low errors and lower overall processing 
time. The SURF descriptor was the more sensitive one to outliers and therefore provided the lower co-registration accuracy. Overall, the algorithm converged in all cases in a few minutes, with the source code written in Matlab ${ }^{\circledR}$ ) allowing real-time application and real-time hypercube registration through an optimized $\mathrm{C} / \mathrm{C}++$ implementation. In particular, the entire process using the SURF algorithm, was completed in about 10-15 min, using the SIFT descriptor was completed in maximum 8 min. The ASIFT descriptor required much more time i.e., from at least 30 to $90 \mathrm{~min}$, depending on the number of the detected inliers. The developed algorithm has been evaluated both qualitatively and quantitative over different datasets, both in rural, agricultural and peri-urban areas. In particular, in agricultural areas errors were larger since in contrast to the structured ones, their smooth structure and texture produce a limited number of correspondences. The experimental results and their validation appear promising. Even with significant spectral variation and important rotation and translation effects, the methodology can deliver enough inliers with adequate accuracy.

\section{Acknowledgments}

We would like to thank the anonymous reviewers for the attentive review. Their comments and suggestions did improve the quality of the paper. This research has been co-financed by the European Union (European Social Fund-ESF) and Greek national funds through the Operational Program "Education and Lifelong Learning" of the National Strategic Reference Framework (NSRF)-Research Funding Program: THALES: Reinforcement of the interdisciplinary and/or inter-institutional research and innovation.

\section{Author Contributions}

Both authors contributed to the design, development and evaluation of the proposed methodology and the writing of the manuscript.

\section{Conflicts of Interest}

The authors declare no conflict of interest.

\section{References}

1. Plaza, A.; Benediktsson, J.A.; Boardman, J.; Brazile, J.; Bruzzone, L.; Camps-Valls, G.; Chanussot, J.; Fauvel, M.; Gamba, P.; Gualtieri, A.; et al. Recent advances in techniques for hyperspectral image processing. Remote Sens. Environ. 2009, 113, S110-S122.

2. Thenkabail, P.; Lyon, G.; Huete, A. Hyperspectral Remote Sensing of Vegetation; CRC Press-Taylor and Francis Group: Boca Raton, FL, USA, 2011; p. 781.

3. Van der Meer, F.; van der Werff, H.; van Ruitenbeek, F.; Hecker, C.; Bakker, W.; Noomen, M.; van der Meijde, M.; Carranza, E.; de Smeth, J.; Woldai, T. Multi- and hyperspectral geologic remote sensing: A review. Int. J. Appl. Earth Observ. Geoinf. 2012, 14, 112-128.

4. Brook, A.; Ben-Dor, E. Advantages of the boresight effect in hyperspectral data analysis. Remote Sens. 2011, 3, 484-502. 
5. Hruska, R.; Mitchell, J.; Anderson, M.; Glenn, N. Radiometric and geometric analysis of hyperspectral imagery acquired from an unmanned aerial vehicle. Remote Sens. 2011, 4, 2736-2752.

6. Zarco-Tejada, P.; Gonzalez-Dugo, V.; Berni, J. Fluorescence, temperature and narrow-band indices acquired from a UAV platform for water stress using a micro-hyperspectral images and a thermal camera. Remote Sens. Environ. 2011, 117, 322-337.

7. Honkavaara, E.; Saari, H.; Kaivosoja, J.; Polonen, I.; Hakala, T.; Litkey, P.; Makynen, J.; Pesonen, L. Processing and assessment of spectrometric, stereoscopic imagery collected using a lightweight UAV spectral camera for precision agriculture. Remote Sens. 2013, 5, 5006-5039.

8. Erivesa, H.; Fitzgeraldb, G. Automated registration of hyperspectral images for precision agriculture. Comput. Electron. Agric. 2005, 47, 103-119.

9. Erivesa, H.; Fitzgeraldb, G.; Clarkec, T. Non-rigid registration of hyperspectral imagery for analysis of agronomic scenes. Biosyst. Eng. 2007, 98, 267-275.

10. Dawn, S.; Saxena, V.; Sharma, B. Remote Sensing Image Registration Techniques: A Survey. In Image and Signal Processing; Elmoataz, A., Lezoray, O., Nouboud, F., Mammass, D., Meunier, J., Eds.; Springer-Berlin: Heidelberg, Germany, 2010; Volume 6134, pp. 103-112.

11. Sotiras, A.; Davatzikos, C.; Paragios, N. Deformable medical image registration: A survey. IEEE Trans. Med. Imag. 2013, 32, 1153-1190.

12. Lowe, D. Distinctive image features from scale-invariant keypoints. Int. J. Comput. Vis. 2004, 20, 91-110.

13. Tuytelaars, T.; Mikolajczyk, K. Local invariant feature detectors: A survey. Found. Trends Comput. Graph. Vis. 2007, 3, 177-280.

14. Bradley, P.; Jutzi, B. Improved feature detection in fused intensity-range images with complex SIFT (CSIFT). Remote Sens. 2011, 3, 2076-2088.

15. Mikolajczyk, K.; Tuytelaars, T.; Schmid, C.; Zisserman, A.; Matas, J.; Schaffalitzky, F.; Kadir, T.; van Gool, L. Internationaljournal of computer vision. Found. Trends Comput. Graph. Vis. 2005, 65, 43-72.

16. Bay, H.; Ess, A.; Tuytelaars, T.; van Gool, L. SURF: Speeded up robust features. Comput. Vis. Image Underst. 2008, 10, 346-359.

17. Morel, J.; Yu, G. ASIFT: A new framework for fully affine invariant image comparison. SIAM J. Img. Sci. 2009, 2, 438-469.

18. Strecha, C.; Bronstein, A.M.; Bronstein, M.M.; Fua, P. LDAHash: Improved matching with smaller descriptors. IEEE Trans. Patt. Anal. Mach. Intell. 2012, 34, 66-78.

19. Yi, Z.; Cao, Z.; Xiao, Y. Multi-spectral remote image registration based on SIFT. Electron. Lett. 2008, 44, 4-5.

20. Aguilera, C.; Barrera, F.; Lumbreras, F.; Sappa, A.; Toledo, R. Multispectral image feature points. Sensors 2012, 12, 12661-12672.

21. Sima, A.; Buckley, S. Optimizing SIFT for matching of short wave infrared and visible wavelength images. Remote Sens. 2013, 5, 2037-2056.

22. Mukherjee, A.; Velez-Reyes, M.; Roysam, B. Interest points for hyperspectral image data. IEEE Trans. Geosci. Remote Sens. 2009, 47, 748-760. 
23. Dorado-Munoz, L.; Velez-Reyes, M.; Mukherjee, A.; Roysam, B. A vector SIFT detector for interest point detection in hyperspectral imagery. IEEE Trans. Geosci. Remote Sens. 2012 , 50, 4521-4533.

24. May, M. Data Analytics and Methods for Improved Feature Selection and Matching. Ph.D. Thesis, The University of Manchester, Manchester, UK, 2012.

25. Vakalopoulou, M.; Karantzalos, K. Automatic Co-Registration of Frame Hyperspectral Data from UAVs. In Proceedings of the ISPRS Workshop on UAV-Based Remote Sensing Methods for Monitoring Vegetation, Cologne, Germany, 9-10 September 2013; pp. 1-6.

26. Sonka, M.; Fitzpatrick, J. Handbook of Medical Imaging. In Medical Image Processing and Analysis; SPIE Press: Bellingham, WA, USA, 2000; pp. 447-515.

27. Makelainen, A.; Saari, H.; Hippi, I.; Sarkeala, J.; Soukkamaki, J. 2D Hyperspectral frame imager camera data in photogrammetric mosaicking. Int. Arch. Photogramm. Remote Sens. Spatial Inf. Sci. 2013, XL-1/W2, 263-267.

(c) 2014 by the authors; licensee MDPI, Basel, Switzerland. This article is an open access article distributed under the terms and conditions of the Creative Commons Attribution license (http://creativecommons.org/licenses/by/3.0/). 